\title{
MAPEANDO LOS PASOS INTEROCEÁNICOS AUSTRALES: LA PRODUCCIÓN Y CIRCULACIÓN DE CONOCIMIENTO HIDROGRÁFICO Y CARTOGRÁFICO EN LAS EXPEDICIONES ESPAÑOLAS A FINES DEL SIGLO XVIII
}

NATALIA GÁNDARA

\section{RESUMEN}

Durante la segunda mitad del siglo XVIII, las exploraciones científico-navales por el océano Pacífico se intensificaron con el fin de reconocer este espacio marítimo y asegurar la mejor ruta de navegación. Como resultado, se incrementó el interés europeo por conocer los pasos interoceánicos que conectan el océano Atlántico con el Mar del Sur. En este contexto, lugares remotos y periféricos como los pasos del estrecho de Magallanes y cabo de Hornos adquirieron un renovado valor para las potencias imperiales. Siguiendo el "giro espacial" en la historia de la ciencia, este artículo tiene por objetivo explorar la producción y circulación de conocimiento hidrográfico y cartográfico acerca de los pasos interoceánicos del estrecho de Magallanes, cabo de Hornos y los canales de la Patagonia occidental en las expediciones españolas entre 1785 y 1795. Se argumenta que la producción de conocimiento es un proceso transimperial que involucra distintos actores. Con ello, se busca problematizar las relaciones de poder que intervienen en los procesos de construcción social de conocimiento, explorando la manera en que la estandarización científica impone una jerarquización sobre las formas de producir y comunicar ciencia. Asimismo, se argumenta que estas expediciones deben comprenderse como parte de un esfuerzo global de la monarquía española por (re)apropiarse de la geografía de los pasos interoceánicos australes en un contexto de intensa competencia imperial por el control de la navegación global.

PALABRAS CLAVE: producción de conocimiento, hidrografía, cartografía, Patagonia.

\section{MAPPING THE SOUTHERN PASSAGES: \\ THE PRODUCTION AND CIRCULATION OF HYDROGRAPHIC AND CARTOGRAPHIC KNOWLEDGE IN THE LATE $18^{\mathrm{TH}}$ CENTURY SPANISH EXPEDITIONS.}

\begin{abstract}
In the second half of the 18th century there was an increase in European naval and scientific expeditions to the Pacific Ocean in order to survey this maritime space and establish the best navigational route. As a result, the connecting passages between the Atlantic and the Pacific Ocean became more
\end{abstract}


relevant. In this context, remote and peripheral places such as the Magellan Strait and Cape Horn gained a new geostrategic value for the European empires. Following the "spatial turn" of the History of Science, this article explores the production and circulation of hydrographic and cartographic knowledge about the southern passages of the Magellan Strait, Cape Horn and the Western Patagonian Channels in the Spanish expeditions between 1785 and 1795. It is argued that the process of production of knowledge is a trans-imperial phenomenon which involves different actors. This perspective allows us to problematise the power relations involved in the process of social construction of knowledge, addressing the issue of how the standardization of the scientific processes impose a hierarchy in the ways of producing and transferring scientific knowledge. Furthermore, the paper argues that these expeditions must be understood as part of the global efforts of the Spanish monarchy to (re)appropriate and take control of the geography of the southern passages in a context of intense imperial competition.

KEY WORDS: production of knowledge, hydrography, cartography, Patagonia. INTRODUCCIÓN

En 1798, bajo el auspicio del ministro de Marina español Juan de Lángara, se publicó la carta titulada Carta Esférica de las costas de la América Meridional desde el paralelo $36^{\circ} 30^{\prime}$ de Latitud S. hasta el cabo de Hornos (...) (Fig. 1), en la que se describe el derrotero seguido por la expedición de Alejandro Malaspina en las costas sudamericanas (1789-1794). La carta, además, comprendía los resultados de las expediciones conducidas por Antonio de Córdoba al estrecho de Magallanes y de Nicolás Lobato a la Patagonia occidental. Este documento cartográfico da cuenta de los esfuerzos de la monarquía española por conocer y mapear los remotos pasos interoceánicos que conectan el Atlántico con el Mar del Sur en un contexto de intensa competencia imperial por generar conocimiento sobre esta estratégica región. Al mismo tiempo, permite un acercamiento hacia la manera en que se

1 Armitage y Bashford, 2014. Pacific Histories. Ocean, Land, People. Palgrave Macmillan, Basingstoke; Ballantyne, 2004. Science, Empire and the European Exploration of the Pacific. Ashgate/Variorum, Aldershot; Buschmann, 2014. Iberian Visions of the Pacific Ocean, 1507-1899. Palgrave Macmillan, Basingstoke; Igler, 2013. The Great Ocean. Pacific Worlds from Captain Cook to the Gold Rush. Oxford University Press, New York; Maroto, 2005. Producing the Pacific. Maps and Narratives of Spanish Exploration (1567-1606). Rodopi, Amsterdam; Matsuda, 2012. Pacific Worlds. A history of Seas, Peoples, and Cultures. Cambridge University Press, Cambridge; Parker, 2018. Pepys Island as a Pacific stepping stone: the struggle to capture islands on early modern maps. The British Journal for the History of Science, 51(4), 659677; Sagredo y Moreno (Eds.), 2014. El Mar del Sur en producía y circulaba el conocimiento hidrográfico y cartográfico a fines del siglo XVIII.

Durante las últimas décadas se ha visto un incremento en el interés historiográfico por el Pacífico, particularmente en el estudio de las expediciones científico-hidrográficas desarrolladas en los siglos XVIII y XIX ${ }^{1}$. En este escenario, la búsqueda del paso por Norteamérica (North-West Passage) ha consignado gran atención por parte de los historiadores, quienes han valorado la relevancia de estas empresas en el contexto de expansión de los imperios europeos hacia el Pacífico². El interés de las expediciones científicas por los pasos australes que conectan los océanos Atlántico y Pacífico, por el contrario, ha sido más escaso, a pesar del impacto científico, político y marítimo que éstas tuvieron para la navegación. Por ello, este artículo tiene por objetivo recentrar la mirada en los procesos de producción de conocimiento sobre la mejor ruta de navegación transoceánica a fines del siglo XVIII, haciendo énfasis en la importancia de los estudios hidrográficos de los

la Historia. Ciencia, expansión y poder en el Pacífico. Dirección de Bibliotecas, Archivos y Museos, Santiago; Williams, 1997. The Great South Sea. English Voyages and Encounters 1570-1750. Yale University Press, New Haven.

2 Day, 1986. Search for the Northwest Passage: An Annotated Bibliography. Garland, New York; Igler, 2013, op. cit.; Bernabeu, 1992. El Pacífico Ilustrado: del lago español a las grandes expediciones. Mapfre, Madrid; Buschmann, 2014, op. cit.; Postnikov, 2004. The Search for a Sea Passage from the Atlantic Ocean to the Pacific via North America's Coast: On the History of a scientific Competition. En Tony Ballantyne (Ed.). Science, Empire and the European Exploration of the Pacific. Ashgate/ Variorum, Aldershot. 


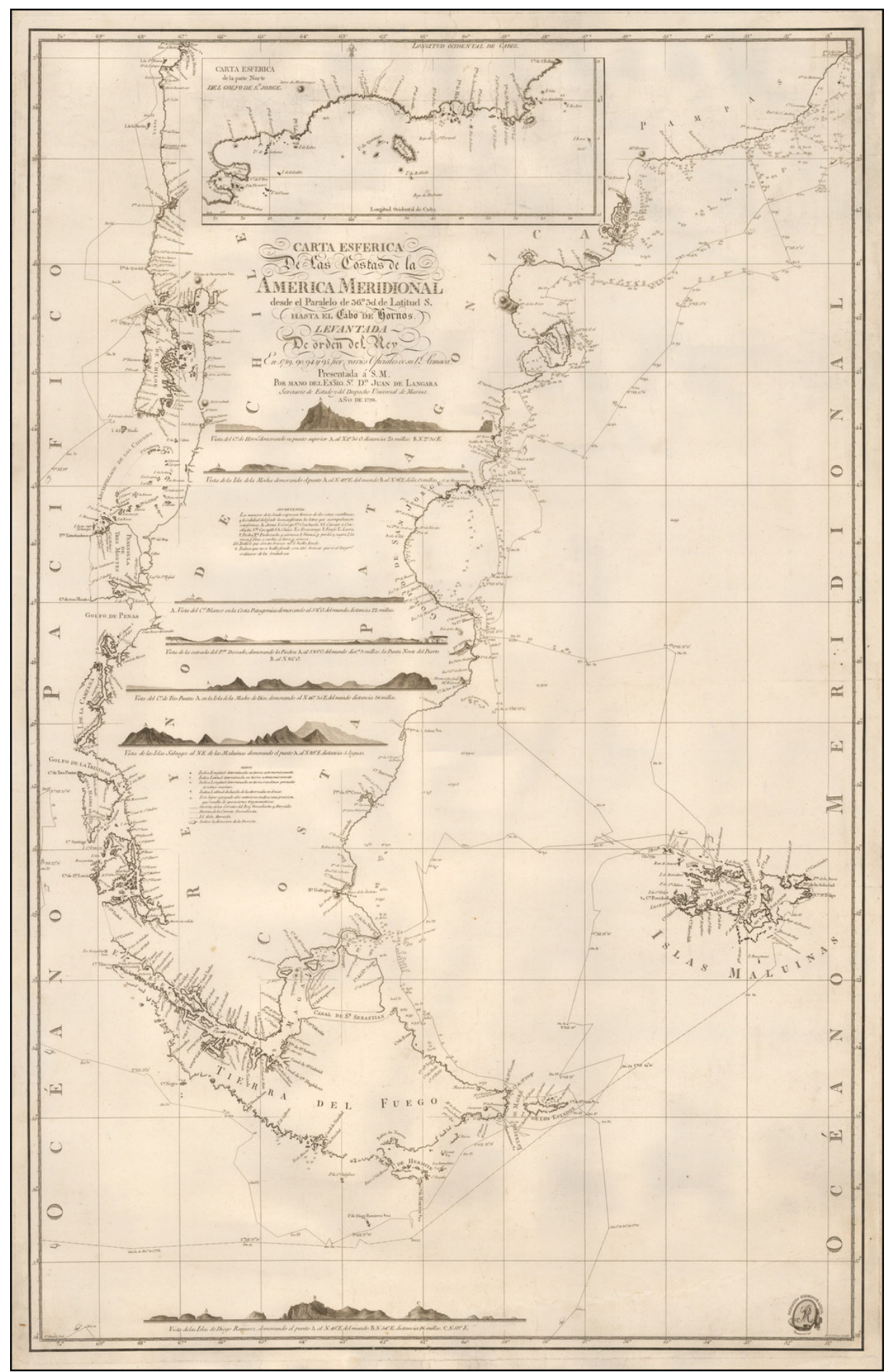

Fig. 1. Juan de Lángara, Carta Esférica de las costas de la América Meridional desde el paralelo 36³0' de Latitud S. hasta el cabo de Hornos (1798). Warren Heckrotte Map Collection, cortesía de Stanford University Libraries https://exhibits.stanford.edu/heckrotte/catalog/xp952vj7734 
pasos interoceánicos del sudoeste (South West passages), a decir, el estrecho de Magallanes, el cabo de Hornos y los canales de la Patagonia occidental.

En los últimos años, la historiografía dedicada a la historia de la ciencia ha reflexionado acerca de las formas de producción, circulación y transferencia de conocimiento ${ }^{3}$. Más aún, el "giro espacial" (spatial turn) en la historia de la ciencia ha ofrecido nuevas formas de pensar estos procesos, resaltando la manera en que las escalas de lo local, regional $y$ global se interconectan ${ }^{4}$. Este enfoque llama a repensar las narrativas eurocéntricas de la historia de la ciencia y analizar las redes que permiten el desarrollo y circulación de conocimiento científico. Un aporte importante de esta perspectiva ha sido la idea de la transferencia de conocimiento científico como un proceso directamente relacionado con las dinámicas espaciales ${ }^{5}$. Esta perspectiva enfatiza las conexiones existentes entre los distintos sitios de conocimiento, lo que a su vez permite estudiar la configuración de las redes de conocimiento transnacionales y transferencia de información, ideas e ideologías a nivel global ${ }^{6}$. Para ello, se analizarán las expediciones imperiales españolas lideradas por Antonio de Córdoba (1786-1789) al estrecho de Magallanes, el análisis de Alejandro Malaspina (1789-1794) sobre la ruta del cabo de Hornos y las expediciones hispanoamericanas conducidas por los pilotos Francisco Clemente y Miró (1792) y José de Moraleda (1793-1795) a la Patagonia occidental.

El artículo argumenta que estas expediciones deben comprenderse como parte de un esfuerzo imperial por reapropiarse de la geografía de los pasos interoceánicos del sudoeste en un contexto de intensa competencia por el control político,

3 Secord, 2004. Knowledge in Transit. Isis, 95(4), pp. 654-672; Cañizares-Esguerra, 2001. How to write the history of the New World: histories, epistemologies, and identities in the eighteenth-century Atlantic world. Standford University Press, Standford; Cañizares-Esguerra y Breen, 2013. Hybrid Atlantics: Future Directions for the History of the Atlantic World. History Compass, 11(8), pp. 597-609; Roberts, 2009. Situating Science in Global History: Local Exchanges and Networks of Circulation. Itinerario, 33(1), 9-30; Sanhueza (Ed), 2017. La movilidad del saber científico en América Latina. Objetos, prácticas e instituciones (siglos XVIII al XX). Editorial Universitaria, Santiago; Davids, 2015. Introduction: Bridging Concepts. marítimo y simbólico de esta región estratégica para la navegación global. Al hacer esto, las expediciones transformaron estos paisajes costeros en laboratorios hidrográficos al medir, describir, mapear y localizar latitudes y longitudes en diversos lugares, generando un cuerpo de conocimiento geográfico e hidrográfico clave e instrumental a los intereses geoestratégicos del imperio español. Asimismo, el artículo discute la producción de conocimiento acerca de esta geografía remota como un proceso transnacional y trans-imperial donde se involucraron distintos actores. Con ello, se busca problematizar las relaciones de poder que intervienen en la construcción social de conocimiento, explorando la manera en que los procesos de estandarización científicos imponen una jerarquización sobre las formas de producir y comunicar los resultados de investigación científica, en este caso hidrográfica y cartográfica. Para ello, las principales fuentes de información serán los diarios de navegación y los diferentes planos y cartas marítimas producidos por estas expediciones.

Los mapas son importantes fuentes para estudiar la relación entre imperio, exploración y ciencia. Sin embargo, tal como lo ha argumentado Katherine Parker, son pocas las investigaciones que han centrado su análisis en estos documentos a pesar de su importancia en la competencia imperial durante el siglo XVIII ${ }^{7}$. La historiografía centrada en los pasos australes ha tratado los mapas como fuentes secundarias dentro de la producción científica de las expediciones. Este artículo, por el contrario, los pondera como documentos centrales del quehacer científico-marítimo de la segunda mitad del siglo XVIII. Los mapas analizados son cartas marinas, es decir, documentos que delinean

Isis, 106(4), pp. 835-839; Achbari, 2015. Building Networks for Science: Conflict and Cooperation in Nineteenth-Century Global Marine Studies. Isis, 106(2), pp. 257-282.

4 McCook, 2013. Introduction. Isis, 104(4), 773-776.

5 Livingstone, 2005. Science, text and space: thoughts on the geography of reading. Transactions of the Institute of British Geographers, 30(4), 391-401.

6 Armitage, 2014. The International Turn in Intellectual History. En Darrin M. McMahon and Samuel Moyn (Eds.). Rethinking Modern European Intellectual History. Oxford University Press, New York, pp. 232-252.

7 Parker, 2018, op. cit., p. 661. 
la hidrósfera con el fin de guiar la navegación oceánica ${ }^{8}$. Estos documentos son resultado de un particular conjunto de prácticas especializadas y la acción de diversos actores involucrados en su producción. Asimismo, el estudio de los mapas permite adentrarse en las dinámicas de transferencia de conocimiento, ya que como objeto, transita entre los contextos locales y las redes transnacionales, estando en permanente tensión y movimiento9. Este artículo explora las dinámicas de producción y circulación de las cartas marinas de los pasos interoceánicos australes a fines del siglo XVIII.

Se dividirá en dos partes. La primera sección abordará el proceso de producción y transferencia de conocimiento hidrográfico sobre los pasos de cabo de Hornos y estrecho de Magallanes producidos a partir de las expediciones de Antonio de Córdoba y Alejandro Malaspina. Esta sección enfatiza la complementariedad de estas expediciones, comprendiéndolas como un esfuerzo de la monarquía española por estudiar y apropiarse nominalmente de este espacio de conexión global. La segunda parte se enfocará en la producción científica desarrollada por las expediciones hispanoamericanas hacia la Patagonia occidental, analizando al mismo tiempo, cómo esta información circuló y se transfirió en las redes de información imperiales y los actores involucrados en esta red de conocimiento.

\section{LA COMPETENCIA IMPERIAL Y LA TRANSFERENCIA DE CONOCIMIENTO SOBRE LOS PASAJES INTEROCEÁNICOS DEL SUDOESTE: ESTRECHO DE MAGALLANES Y CABO DE HORNOS}

A partir del viaje de circunnavegación de George Anson a principios de la década de 1740,

8 Edney, 2019. Cartography. The ideal and its history. The University of Chicago Press, Chicago, p. 3.

9 Lois, 2014. Mapas para la Nación. Episodios en la historia de la cartografía argentina. Biblios Ed., Buenos Aires.

10 Álvarez, 2010. Las conexiones entre el pensamiento de Alejandro Malaspina y la representación visual de la expedición en la Patagonia (1789-1794). Magallania, 38(1), pp. 5-18; Estefane, 2005. La proyección nacional de una empresa imperial: la expedición Malaspina (1789-1794) en Chile republicano. Historia, 38(2), 287-326; Martinic, el Mar del Sur se abrió a una competencia entre las potencias imperiales europeas por generar conocimiento náutico y cartográfico sobre esta región. En la medida que el tráfico comercial y el interés geopolítico de los imperios europeos se intensificaban en el Pacífico, las expediciones científico-navales por estas aguas se hicieron más comunes y necesarias para la navegación eficiente y segura. En este contexto, los imperios europeos, principalmente Gran Bretaña, España y Francia, dedicaron grandes esfuerzos por generar conocimiento en áreas claves, siendo particularmente relevante la relativa a los pasos interoceánicos que conectan el Atlántico con el Pacífico. De este modo, los remotos pasos de conexión del estrecho de Magallanes y cabo de Hornos adquirieron mayor relevancia para la navegación global, estableciéndose una discusión entre los exploradores del periodo por determinar cuál era la mejor ruta hacia el Mar del Sur. Por un lado, estaban los exploradores como Louis Antoine de Bougainville, que asignaban la ruta magallánica como la mejor manera de atravesar de un océano al otro; mientras que otros, como James Cook, preferían la vía por el cabo de Hornos. Como parte de las redes científico-navales globales, el imperio español envió diferentes expediciones a fines del siglo XVIII para determinar cuál era el mejor paso interoceánico y con ello generar una ruta segura y eficaz para la navegación militar y comercial. Estas fueron las expediciones de Antonio de Córdoba, quien exploró las costas interiores para determinar la viabilidad del paso por el estrecho de Magallanes, mientras que la expedición liderada por Alejandro Malaspina estudió la ruta por mares abiertos, navegando a través del cabo de Hornos.

Tradicionalmente, la historiografía ha analizado por separado estas dos expediciones ${ }^{10}$. Sin embargo, una mirada en conjunto enriquece

2004. Archipiélago Patagónico. La última frontera. Ediciones de la Universidad de Magallanes, Punta Arenas; Pimentel, 1989. Malaspina y la Ilustración. Ministerio de Defensa, Madrid; Pimentel, 1994. Ciencia y Política en el pensamiento colonial de Alejandro Malaspina (1754 1794). Universidad Complutense de Madrid, Madrid; Riera, 1988. Carlos III y el control del estrecho de Magallanes. La expedición del capitán de navío don Antonio de Córdoba (1785-1786). 2 $2^{\circ}$ Congreso de Historia Militar. Colección ADALID, Servicio de Publicaciones del EME, Tomo III, Comunicaciones II, Zaragoza, pp. 101-112; Sagredo 
el análisis sobre los esfuerzos de la monarquía española por conocer y controlar zonas geográficas claves para la navegación transoceánica a fines del siglo XVIII. Al mismo tiempo, este análisis permite resaltar la importancia, utilidad $e$ instrumentalidad de las expediciones hidrográficas a la consolidación del imperialismo en los mares globales. Solo recientemente investigadores como D. Graham Burnett, Jason W. Smith y Katherine Anderson han recalcado la relevancia de la práctica de la ciencia hidrográfica como un aspecto central en el sistema de competencia imperial a nivel global en el siglo XIX para los casos de Estados Unidos y Reino Unido, respectivamente ${ }^{11}$. Siguiendo estos argumentos es importante estudiar las expediciones hidrográficas, pues ellas permiten analizar históricamente las relaciones inter-imperiales a fines del siglo XVIII y comprender el proyecto global del imperio español por retener el dominio político y simbólico del océano Pacífico y el Atlántico sur.

Durante el siglo XVIII y la primera mitad del siglo XIX, las expediciones hidrográficas se enfocaron en estudiar las costas planetarias, midiendo las aguas costeras en canales, estrechos, fiordos, ensenadas y bahías en islas y continentes, además de cartografiar y georeferenciar estos lugares. Adicionalmente, las expediciones hidrográficas se dedicaban a descubrir, describir y evaluar diferentes rutas de navegación, estudiando los vientos, corrientes, clima y temperaturas oceánicas y continentales. Tal como lo explica Helen Rozwadowski, durante

y González (Eds.), 2004. La expedición de Malaspina en la frontera austral del imperio español. Editorial Universitaria y Centro de Investigaciones Diego Barros Arana, Santiago; Vázquez de Acuña, 1998. La expedición científico-política del Capitán de Navío Don Alejandro Malaspina (1789-1794). Boletín de la Academia Chilena de la Historia, 65(108), pp. 133-174; Vázquez de Acuña, 2004. Las exploraciones del estrecho de Magallanes por el capitán de navío don Antonio de Córdoba y Lasso de la Vega. Su primer viaje (1785-1786); su segundo viaje (1788-1789). Revista de Historia Naval, 84, 7-26.

11 Burnett, 2009. Hydrographic Discipline among the Navigators: Charting an 'Empire of Commerce and Science' in the Nineteenth-Century Pacific. En James Akerman (Ed.). The Imperial Map: Cartography and the Mastery of Empire. University of Chicago Press, Chicago; Smith, 2018. To Master the Boundless Sea: The U.S. Navy, the Marine Environment, and the Cartography of Empire. University of North Carolina Press, Chapel Hill; Anderson, este periodo el interés de las ciencias marítimas se centraba en las aguas costeras, cuestión que solo comenzó a cambiar a mediados del siglo $\mathrm{XIX}$, cuando las investigaciones de la incipiente ciencia oceanográfica se trasladaron hacia las profundidades del océano ${ }^{12}$. Las expediciones de Córdoba y Malaspina siguieron los cánones de las exploraciones científicas del periodo, estudiando las costas más meridionales del continente americano, desarrollando cartas hidrográficas de la costa y planos de las diversas bahías e islas australes. Asimismo, éstas documentaron con detalle la ruta seguida y otros datos relevantes como la dirección de los vientos y las condiciones para la navegación.

El primer viaje de Antonio de Córdoba al estrecho de Magallanes tuvo como objetivo explorar las costas patagónicas, estudiar la eficiencia de la navegación a través de este paso interoceánico y evaluar la posibilidad de crear un asentamiento en esta región ${ }^{13}$. A bordo de la fragata Santa María de la Cabeza y con una tripulación de 276 hombres, Córdoba zarpó el día 9 de octubre de 1785 hacia costas americanas. El segundo viaje estuvo motivado por el deseo de generar un conocimiento más riguroso y acabado sobre la entrada occidental del canal, particularmente la zona comprendida entre el cabo Lunes, Pilares y Victoria ${ }^{14}$. Después de alcanzar la entrada oriental del Estrecho a mediados de diciembre, la expedición pasó dos meses reconociendo las costas magallánicas. Por su parte, el análisis de la ruta por mares abiertos fue hecho por la empresa de circunnavegación de

2018. Reading and writing the scientific voyage: FitzRoy, Darwin and John Clunies Ross. The British Journal for the History of Science, 51(3), 369-394.

12 Rozwadowski, 2008. Fathoming the ocean: the discovery and exploration of the deep sea. Belknap, Cambridge, Mass.; London, p. 9

13 Vargas Ponce, 1788. Relación del último viaje al estrecho de Magallanes de la fragata del S.M. Santa María de la Cabeza en los años de 1785 y 1786. Extracto de todos los anteriores desde su descubrimiento impresos y mss. $y$ noticia de los habitantes, suelo, clima y producciones del Estrecho. Viuda de Ibarra, Hijos y Compañía, Madrid, p. xi.

14 Vargas Ponce, 1793. Apéndice a la Relación del viage al Magallanes de la fragata de guerra Santa María de la Cabeza, que contiene el de los paquebotes Santa Casilda y Santa Eulalia para completar el reconocimiento del estrecho en los años de 1788 y 1789. Imprenta de la Viuda de D. Joaquín Ibarra, Madrid, p. 1. 
Alejandro Malaspina. A bordo de las corbetas la Descubierta (comandada por Alejandro Malaspina) y la Atrevida (comandada por José de Bustamante y Guerra), la expedición zarpó desde Cádiz el día 30 de julio de 1789. Con el objetivo de estudiar y evaluar la eficacia de esta ruta, se estudiaron las costas de la Patagonia oriental, Tierra del Fuego y lugares claves de la costa patagónica occidental, tales como el cabo Pilares y las islas Evangelistas, Madre de Dios y Guafo.

La historiografía ha señalado a la expedición de Córdoba como una de las empresas científicas más importantes en el reconocimiento del estrecho de Magallanes. En particular, se ha destacado sus contribucionesala cartografía, hidrografía, geografía y etnografía de la región ${ }^{15}$. Más recientemente, los estudios de Vázquez de Acuña y Buschmann han integrado la expedición de Córdoba en el marco de reformación, profesionalización y renovación de las ciencias náuticas españolas ${ }^{16}$. Por su parte, la expedición de Malaspina ha sido caracterizada como la contraparte española de los viajes de exploración imperial realizados por Cook y La Pérouse ${ }^{17}$. A partir del trabajo de Pimentel, la historiografía se ha concentrado en resaltar la importancia de la expedición comandada por Malaspina, particularmente enfatizando sus contribuciones a las ciencias naturales, la etnografía, la geografía, entre otros, al conocimiento del mundo del Pacífico y las posesiones coloniales españolas en América ${ }^{18}$. Más aún, tanto las exploraciones de Córdoba como el viaje de circunnavegación de Malaspina deben enmarcarse en lo que Manuel Sellés ha denominado la emergencia de la navegación científica ${ }^{19}$. De este modo, la historiografía ha reconocido el valor de esta geografía y de las exploraciones de forma pormenorizada. No obstante, si se toma en cuenta el punto de vista de los estudios hidrográficos de la época, este espacio adquiere integridad territorial. No son solamente las frías aguas $y$ costas patagónicas y fueguinas, sino un laboratorio

15 Riera, 1988, op. cit.; Vázquez de Acuña, 2004, op. cit.

16 Buschmann, 2014, op. cit.; Vázquez de Acuña, 2004, op. cit.

17 Vázquez de Acuña, 1998, op. cit.

18 Pimentel, 1989, op. cit.; Pimentel, 1994, op. cit.; Buschmann, 2014, op. cit.; Sagredo, 2013. De la hidrografía imperial a la hidrografía nacional. Reconocimientos del Pacífico Sur. Siglos XVIII y XIX. Anuario de Estudios Americanos, 70(2), 509-556; Bernabeu, 1992, op. cit.; hidrográfico donde las expediciones dan vida a un territorio al reconocer y cartografiar la zona y al aplicar nuevas técnicas e instrumentos para generar un conocimiento científico y riguroso de la geografía costera.

La producción de conocimiento hidrográfico estuvo en el centro de estas empresas científiconavales. El material cartográfico legado por estas expediciones da cuenta del detalle y rigurosidad del trabajo hidrográfico. Por ejemplo, la expedición de Córdoba pasó tres meses estudiando científicamente las costas del estrecho de Magallanes. La Fig. 2, titulada Tres bahías del Estrecho (...) (1786), retrata los esfuerzos de la expedición por medir y mapear los principales accidentes geográficos de él. La idea general era evaluar los lugares más seguros para fondear naves. Cada uno de estos planos muestra la profundidad de estas diversas bahías, así como también su ubicación en latitud y longitud. En la misma lógica, la expedición de Malaspina reconoció y mapeó con rigurosidad lugares estratégicos de la geografía austral, los cuales eran evaluados para ser usados como lugares de paso para descansar y reponer a la tripulación. De esta forma, la expedición produjo cartas marinas de diferentes puertos, islas y bahías, particularmente en la costa atlántica: San José, San Gregorio, Santa Elena, Puerto Deseado, San Julián, Santa Cruz, el río Gallegos y el Puerto Egmont en las Falklands, el de Año Nuevo en la isla de los Estados y la bahía Buen Suceso en Tierra del Fuego ${ }^{20}$.

$\mathrm{Al}$ desarrollar sus exploraciones, las expediciones lideradas por Córdoba y Malaspina no solo produjeron nuevo conocimiento sobre la geografía patagónica y fueguina, sino que éste entró en diálogo con las expediciones europeas que las precedieron. En particular, el análisis detenido de la cartografía desarrollada por estas expediciones da cuenta de las redes transnacionales que operaron en la construcción de conocimiento hidrográfico en el siglo XVIII.

Ballantyne, 2004, op. cit.; Maeso, 2005. Expediciones navales españolas a la Patagonia argentina durante el siglo XVIII. Universidad Nacional de Educación a Distancia, Madrid.

19 Sellés, 2000. Navegación astronómica en la España del siglo XVIII. Universidad Nacional de Educación a Distancia, Madrid.

20 Sagredo y González (Eds.), 2004, op. cit., p. 169. Vargas Ponce, 1788, op. cit., p. 118. 


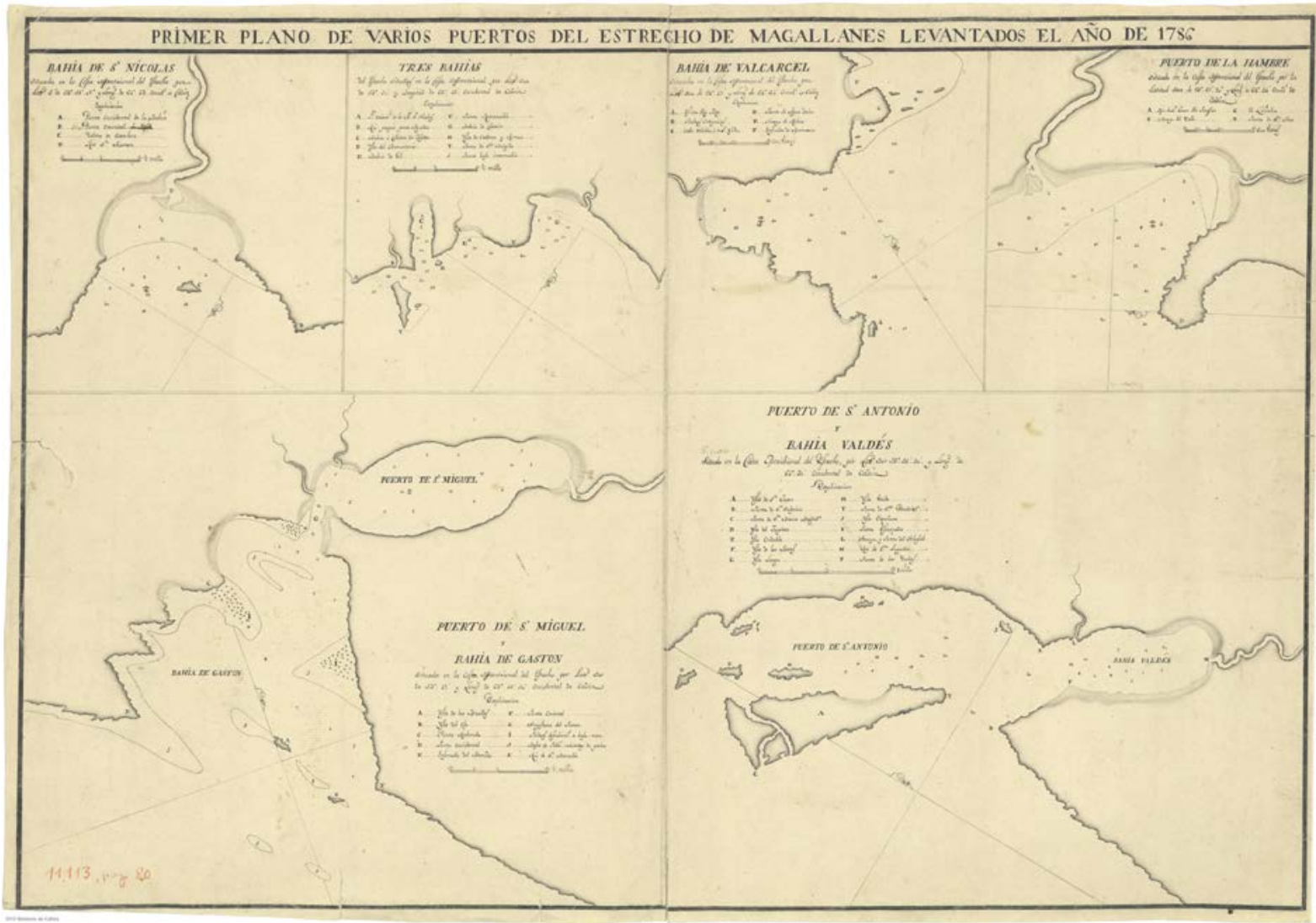

Fig. 2. Anónimo, Tres bahías del Estrecho: situadas en la Costa Septentrional por Latitud Sur de 53 ${ }^{\circ} 50^{\prime}$ y Longitud de $66^{\circ} 43^{\prime}$ occidental de Cádiz. Primer plano de varios puertos del estrecho de Magallanes levantados el año de 1786 (1786). Museo Naval de Madrid MN 53-C-9.

Por ejemplo, la Carta Reducida del estrecho de Magallanes (...) (Fig. 3), producida a partir del primer viaje de Córdoba, demuestra cómo los resultados de esta empresa se unieron al conocimiento $e$ información prexistente sobre la geografía magallánica. Tal como se explica en la inscripción de la Carta, se tomaron datos de los mapas y diarios de navegación de otras expediciones. Así, por ejemplo, la parte oriental de la Tierra del Fuego está copiada del mapa que se incluyó en la publicación española del viaje de John Byron alrededor del mundo, mientras que la parte oriental del continente y Tierra del Fuego fue trabajada a partir de las cartografías del segundo viaje de James Cook. Asimismo, los autores de la Carta advierten que esta información fue comparada y visada con los datos de las expediciones españolas de los hermanos Nodal y Sarmiento. De este modo, se debe sobrepasar la mirada nacionalista que encaja estas expediciones dentro del marco de renovación imperial, y comprender cómo estas empresas fueron parte de una red científica a escala global.

Tal como lo demuestra la expedición de Antonio de Córdoba al estrecho de Magallanes, la producción y transferencia de conocimiento hidrográfico y geográfico en la segunda mitad del siglo XVIII fue un proceso activo que no solo consistió en la generación de conocimiento nuevo, sino, muy importante, significó contrastar, comparar y criticar información proveniente de otras empresas científicas. Como se da cuenta en el diario del viaje publicado en 1788, la tripulación de la Santa María de la Cabeza fue corroborando y cotejando los datos levantados por previas expediciones. Por ejemplo, se criticó la información cartográfica 
de Bougainville, quien se habría equivocado en la posición del eje del norte en su mapa ${ }^{21}$, así como la información levantada por el capitán Cook, quien había ubicado la entrada oriental del Estrecho 24' más al oriente ${ }^{22}$. Asimismo, el editor de la obra, José de Vargas Ponce (1760-1821), adaptó y editó información de las expediciones anteriores en aquellas partes del Estrecho donde la tripulación de Córdoba no pudo hacer un reconocimiento detallado, con el fin de conseguir una completa representación de la geografía magallánica. En particular, la expedición no pudo realizar el reconocimiento hidrográfico en la parte occidental del Estrecho debido a las inclemencias climáticas y otros factores ambientales. De esta manera, Vargas Ponce tomó los datos faltantes de la investigación del inglés Samuel Wallis y completó la descripción de la Patagonia occidental con las informaciones de Hawkesworth, Boungainville y Juan y Ulloa, entre otros ${ }^{23}$. Sin embargo, al juntar la información de estas diversas fuentes, se creó una imagen distorsionada y confusa de la geografía magallánica, particularmente en su lado occidental. Por ello, Vargas Ponce recomendó realizar otra exploración enfocada en el reconocimiento occidental del Estrecho y estudios adicionales acerca de la Patagonia occidental. Esta segunda expedición zarpó en los paquebotes Santa Eulalia y Santa Casilda y llevó a los jóvenes oficiales Cosme de Churruca y Ciriaco de Cevallos, quienes corrigieron e hicieron nuevos cálculos astronómicos y observaciones geográficas de la costa

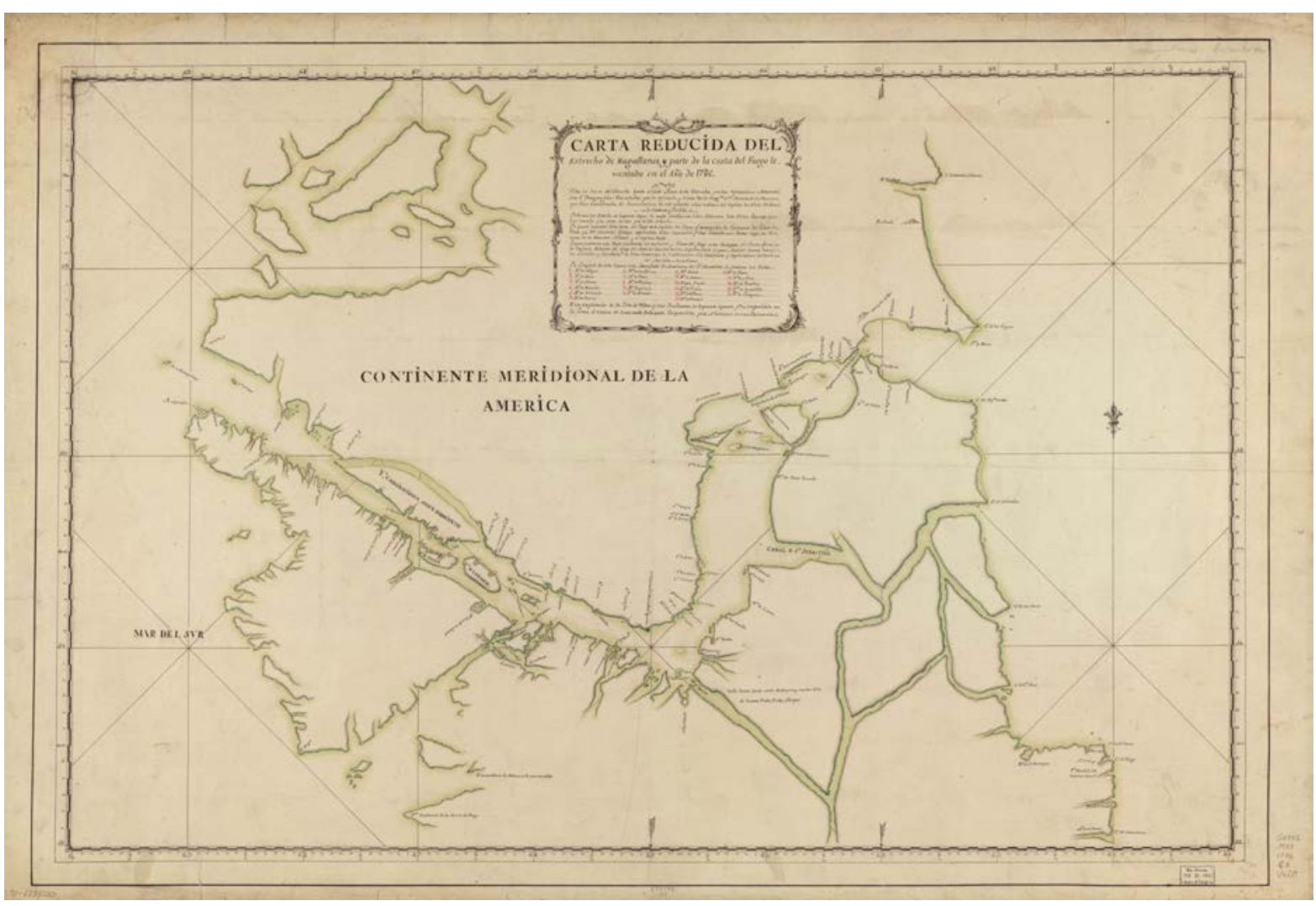

Fig. 3. Anónimo, Carta Reducida del estrecho de Magallanes, y de la parte de la Costa del Fuego levantado en el año 1786 (1786).

Library of Congress Geography and Map Division Washington, D.C. 20540-4650

USA dc: https://www.loc.gov/resource/g5332m.ct000276/

21 Vargas Ponce, 1788, op. cit., p. 118.

22 Ibidem, p. 152.
23 Ibidem, p. 48. 
magallánica. Tal como en el primer viaje, los resultados del segundo fueron dados a conocer en un volumen titulado Apéndice a la Relación del viage de Magallanes (...), publicado en 1793. Este breve volumen fue desarrollado con un enfoque más pragmático, enfatizando el lenguaje y la finalidad científica de la segunda expedición, presentando la descripción de la ruta seguida por los paquebotes, así como también los resultados de las mediciones hidrográficas y observaciones astronómicas y geográficas.

Las conclusiones alcanzadas por las expediciones de Córdoba tuvieron importantes implicancias para la navegación por los mares australes. De acuerdo a la evaluación de la tripulación, se determinó que el estrecho de Magallanes no era el paso más adecuado para atravesar de un océano al otro. En el diario de navegación se argumentó que ese paso no debía seguirse, y ni siquiera considerarse, pues los diversos elementos naturales como los vientos, corrientes, el fondo y la estrechez de los canales representaban obstáculos insuperables, impidiendo la travesía segura de las embarcaciones ${ }^{24}$. De forma importante, las conclusiones alcanzadas por el equipo comandado por Córdoba vinieron a integrar el debate por determinar la mejor ruta interoceánica por los mares australes durante la segunda mitad del siglo XVIII. Louis Antoine de Bougainville, por ejemplo, sostenía que, a pesar de las dificultades de la navegación por el estrecho de Magallanes, ésta era preferible en los meses de verano ${ }^{25}$. Los oficiales y exploradores británicos John Byron y James Cook, por otro lado, argumentaban que la navegación por la ruta magallánica era más arriesgada y difícil, aunque Byron sostenía que podía realizarse en la temporada adecuada ${ }^{26}$. Siguiendo la tradición española, particularmente el diagnóstico de los hermanos Nodal, Córdoba determinó que la razón y la experiencia hacían desestimar la ruta del Estrecho y con ello considerar preferible la vía del cabo de Hornos por ser menos riesgosa y azarosa ${ }^{27}$. Con esta evaluación, Córdoba

24 Ibidem, p. 46.

25 Ibidem, p. 162.

26 Idem, p. 162; Cook, 1893. Captain Cook's Journal during his first voyage round the world made in H.M. Bark "Endeavour" 1768-1771. Elliot Stock, London, pp. 47-48; Byron, 1769. Viage del Comandante Byron cerró la posibilidad de usar este paso como canal conector entre los dos océanos.

Los argumentos esgrimidos por la expedición de Córdoba fueron respaldados por Alejandro Malaspina. Más aún, éste consideró que los resultados de Córdoba venían a formar parte de una red de investigaciones hidrográficas europeas que avalaban la idea de que el paso por el Estrecho no era eficiente para la navegación interoceánica. De este modo, y a pesar de que las instrucciones sostenían la necesidad de investigar toda la costa patagónica, Malaspina en conjunto con sus oficiales, decidieron no adentrarse por el Estrecho. En su diario de navegación argumentó la futilidad de otra expedición al Estrecho, evidenciando que la zona ya había sido explorada, reconocida y satisfactoriamente mapeada por Antonio de Córdoba y James Cook ${ }^{28}$. Con el fin de ahorrar tiempo y usar eficientemente sus recursos, el oficial consideró más importante para la navegación y el conocimiento de la zona continuar con la expedición, reconociendo y mapeando con precisión la ruta de navegación exterior, usando la vía del cabo de Hornos.

Con todo, Malaspina se vio en la tarea de determinar cuál era la mejor vía para navegar por el paso del cabo de Hornos, porque había dos rutas principales. La primera se hacía bordeando la costa de Tierra del Fuego, y la segunda, navegando por mares abiertos rodeando el cabo de Hornos. Tomando en consideración su propia experiencia y cotejando los datos de expediciones anteriores, particularmente los viajes del capitán James $\mathrm{Cook}^{29}$, concluyó que la manera más eficiente y segura de navegar era la ruta por mares abiertos. Al determinar este derrotero como el mejor paso, Malaspina se unió a las conclusiones de Córdoba, desalentando la navegación por el estrecho de Magallanes y promoviendo la navegación por mar abierto, tal como había concluido James Cook tras su segundo viaje alrededor del mundo.

$\mathrm{Al}$ igual que para la expedición de Córdoba, la circulación de las obras de otros navegantes europeos es vital para comprender cómo la empresa

alrededor del mundo, hecho últimamente de orden del almirantazgo de Inglaterra. Casa de don Francisco Mariano Nipho, Madrid, p. 142.

27 Vargas Ponce, 1788, op. cit., p. 163.

28 Sagredo y González (Eds.), 2004, op. cit., p. 194 y p. 342

29 Ibidem, p. 190. 
hidrográfica conducida por Malaspina produjo conocimiento sobre la geografía patagónica. En su navegación por los mares americanos australes, éste leyó y usó críticamente los diarios de Sarmiento de Gamboa, del explorador francés Amadée Francois Frezier y del inglés James Cook ${ }^{30}$. Esta información fue también complementada por el conocimiento emanado de las expediciones hispanoamericanas a las costas meridionales del continente americano. Por ejemplo, en su exploración de la costa patagónica occidental, desde el paralelo $57^{\circ}$ sur hasta el archipiélago de Chiloé, Malaspina utilizó y contrastó la información hidrográfica y cartográfica levantada por los viajeros ingleses; de Córdoba, quien informó específicamente sobre los mares adyacentes a la entrada occidental del estrecho de Magallanes; y las expediciones hispanoamericanas a los canales patagónicos, particularmente la información desarrollada por las exploraciones de Francisco de Machado (1768-1769) y José de Moraleda ${ }^{31}$.

Más aún, el análisis de la cartografía desarrollada por la expedición refuerza la idea de la importancia de la circulación de información para la producción de conocimiento hidrográfico y cartográfico. Así, por ejemplo, la Carta Esférica de una parte del Reyno de Chile (...) (Fig. 4) da cuenta de redes existentes en la formación de nuevo conocimiento cartográfico. La carta representa los resultados de la investigación hidrográfica desarrollada por la expedición, desde el golfo de Penas hasta la desembocadura del río Toltén, pero, además, el documento contiene una carta añadida posteriormente donde se copian los resultados de la expedición a los canales patagónicos occidentales liderada por Nicolás Lobato en 1792. Esto es fundamental para la comprensión de los procesos de producción de conocimiento ya que demuestra la activa participación de diversos actores en las redes de la ciencia global. Más aún, esta perspectiva difumina las diferencias entre ciencia metropolitana y ciencia colonial, comprendiendo que estos conocimientos circulaban y entraban en diálogo entre sí.

La publicación de esta carta de navegación,

$30 \quad$ Ibidem, p. 190 y p. 200.

Ibidem, p. 204.

Buschmann, 2014, op. cit., p. 196.

33 Vargas Ponce, 1820. Voyage of discovery to the Strait of en particular, y de los diarios de navegación de Córdoba al estrecho de Magallanes, en general, representó un cambio importante en la manera en que la corona española producía y comunicaba los resultados de sus exploraciones. Desde mediados del siglo XVIII, los escritores españoles habían tomado tímidos pasos para publicar las hazañas exploratorias españolas ${ }^{32}$. El editor de la obra, José de Vargas Ponce, llevó esta idea a otro nivel, juntando en un volumen todas las experiencias de navegación por el Estrecho. Al hacer esto, conectó los viajes españoles del siglo XVI y XVII con los viajes de los exploradores ingleses y franceses de mediados del siglo XVIII, presentando, al mismo tiempo, la expedición de Córdoba dentro de una larga tradición de exploraciones navales españolas. Asimismo, al publicar las conclusiones de la expedición, se buscaba que los resultados obtenidos circularan por las redes de información hidrográfica, legitimándose así la información contenida en la obra. Como lo ha sostenido Matthew Franco, las copias de los volúmenes publicados por Vargas Ponce circularon ampliamente por las redes de conocimiento global. Esto se ve respaldado, además, por la traducción de la obra en Inglaterra, la que fue publicada en Londres en $1820^{33}$.

En suma, las expediciones comandadas por Córdoba y Malaspina hacia los pasos interoceánicos australes del estrecho de Magallanes y el cabo de Hornos representan y ejemplifican las transformaciones de la ciencia náutica española a fines del siglo XVIII. Dan cuenta de los esfuerzos por reformar la manera en la cual se produce y se comunica el conocimiento científico en un contexto de intensa rivalidad imperial. En particular, demuestran que estas empresas, aunque con fuerte carácter y objetivos nacionales, estuvieron en diálogo constante con las expediciones que les precedieron. Más aún, el análisis detenido a la cartografía resultante de la expedición de Malaspina da cuenta cómo ésta se alimentó de las empresas hidrográficas locales (organizadas desde Chiloé) y cómo estos resultados fueron integrados y apropiados por la expedición metropolitana. La siguiente sección analiza los aportes de las

Magellan: with an account of the manners and customs of the inhabitants, and of the natural productions of Patagonia undertaken, by order of the King of Spain. Phillips, London. 


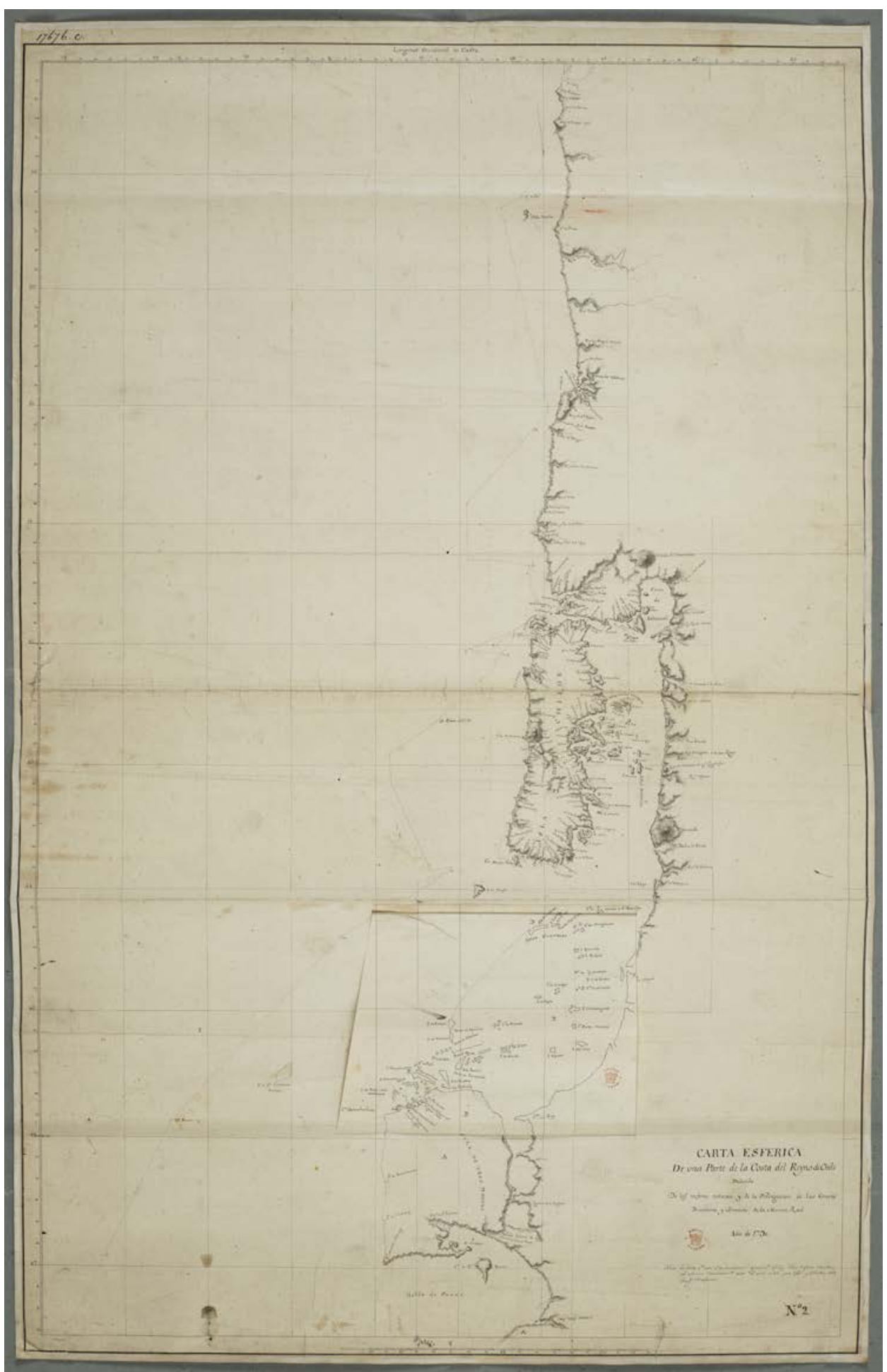

Fig. 4. Anónimo, Carta Esférica de una parte del Reyno de Chile, deducida de las mejores noticias y de la navegación de las corbetas Descubierta y Atrevida de la Marina Real (1790). The British Library, Add MS 17676 C, cortesía de la (c) British Library Board. 
expediciones hispanoamericanas hacia los canales interiores de la Patagonia occidental. A fines del siglo XVIII, esta región permanecía como un área muy poco explorada y se creía que una exploración más detallada podría resultar en el descubrimiento de una ruta más eficiente y fácil para la navegación interoceánica.

\section{PRODUCCIÓN Y CIRCULACIÓN DE CONOCIMIENTO SOBRE LA PATAGONIA OCCIDENTAL. UNA MIRADA DESDE LA HISTORIA GLOBAL}

Existe un riesgo importante al analizar separadamente las expediciones a la Patagonia y los pasos interoceánicos del sudoeste en expediciones locales y metropolitanas. Ello, por ejemplo, podría repercutir en separar arbitrariamente los resultados de estas empresas sin lograr evidenciar a cabalidad la manera en que la información que surgía de estos viajes circulaba dentro de las redes de conocimiento e información del imperio español. Sin embargo, por razones analíticas este artículo las divide con el fin de enfatizar la importancia de la contribución de estas expediciones menores de origen local al acervo del conocimiento hidrográfico y geográfico americano. Lo anterior es relevante debido a que estas expediciones son ignoradas o tratadas muy superficialmente en los análisis históricos del imperio español a las costas del océano Pacífico. Como ha destacado Buschmann, cualquier visión del Pacífico debe considerar también las realidades del imperio español en las colonias americanas $^{34}$. Para este historiador, la conexión de Hispanoamérica con el Pacífico es lo que ha hecho la visión ibérica única y original, particularmente

34 Buschmann, 2014, op. cit., p. 9.

35 Ibidem, p. 20.

36 Ardash, 2012, El Pacífico Hispanoamericano. Politico y Comercio En El Imperio Español (1680-1784). El Colegio de México, Ciudad de México, p. 15.

37 Hanisch, 1982. La isla de Chiloé, capitana de rutas australes. Academia Superior de Ciencias Pedagógicas de Santiago, Santiago.

38 Urbina, 2010. La navegación por los canales australes en la Patagonia Occidental Insular en los siglos coloniales: la ruta de istmo de Ofqui. Magallania, 38(2), 41-67; 2013. Expediciones a las costas de la Patagonia Occidental en el Período Colonial. Magallania, 41(2), 51-84; 2014. El frustrado fuerte de Tenquehuen en el archipiélago de cuando se compara con las representaciones imperiales de Francia y Gran Bretaña ${ }^{35}$. Tal como lo ha sostenido Mariano Ardash, desde una perspectiva económica y comercial, durante los siglos XVII y XVIII el Pacífico se convirtió en un lago indiano ${ }^{36}$. Esta idea se vuelve incluso más relevante cuando se refiere a los reconocimientos hispanos del Pacífico sur, particularmente a la Patagonia y los pasos interoceánicos.

Durante el periodo colonial, el reconocimiento sobre la Patagonia y el estrecho de Magallanes fue fundamentalmente conferido a los gobiernos locales, principalmente a Chiloé, Buenos Aires y Montevideo. Tal como lo ha sostenido Walter Hanisch, Chiloé se convirtió en la capitana de las rutas australes, creando conocimiento sobre la geografía patagónica, nuevas rutas y derroteros para navegar en el mar interior ${ }^{37}$. Esta sección del artículo explorará las expediciones científicas hidrográficas a la Patagonia occidental organizadas por las autoridades de Lima, Santiago y Chiloé, con el fin de enfatizar el rol de estas expediciones en la generación de conocimiento del Pacífico sur y los mares australes, y cómo esta información circuló y fue transferida en las redes de conocimiento global.

Tal como lo ha expuesto Ximena Urbina en sus numerosas publicaciones ${ }^{38}$, los territorios y mares al sur del archipiélago de Chiloé eran considerados como una extensión de esta gobernación; por ello, durante los siglos XVII y XVIII, las autoridades isleñas organizaron una serie de expediciones y avanzadas de carácter militar y religioso. Durante la segunda mitad del siglo XVIII, estas expediciones esporádicas y de carácter coyuntural se volvieron más comunes debido a la sospecha de presencia británica en las costas australes. Por ejemplo, tras la ocupación

los Chonos, 1750: La dimensión chilota de un conflicto hispano-británico. Historia, 47(1), 133-155; 2016. La sospecha de ingleses en el extremo sur de Chile, 16691683: Actitudes imperiales y locales como consecuencia de la expedición de John Narborough. Magallania, 44(1), 15-40; 2017. La isla Madre de Dios (costa del Pacífico austral) en los siglos XVII y XVIII: circulación de la información e intereses geopolíticos de España e Inglaterra. Vegueta, 17, 545-567; 2018b. Los 'papeles de Londres' y alertas sobre ingleses. Chiloé y las costas de la Patagonia Occidental ante los conflictos entre España e Inglaterra: siglos XVII y XVIII. Mélanges de la Casa de Velázquez, 48(2), 235-264. 
británica de Port Egmont en las islas Falklands, entre los años 1767 y 1770 se enviaron tres pequeñas expediciones para explorar, reconocer y mapear los canales de la Patagonia occidental. De acuerdo a Mateo Martinic, se temía especialmente una ocupación en la isla Madre de Dios por su situación casi pegada al continente, por su cercanía al Estrecho, por la extensión de la isla y por las posibilidades que les daría (a los británicos) su posesión ${ }^{39}$. Estas tres expediciones fueron lideradas por Pedro de Mansilla (diciembre 1767- mayo 1768), José de Sotomayor y Francisco Machado (diciembre 1768-mayo 1769) y Joseph Rius (noviembre de 1770-diciembre de 1770). Con estos viajes exploratorios se consiguió recolectar información y crear conocimiento acerca de los canales patagónicos, generando nuevas noticias acerca de las condiciones climáticas y medioambientales de la zona e información sobre las comunidades indígenas. En particular, ellas dieron cuenta de las mejores rutas para la navegación en los canales patagónicos occidentales, describiendo los lugares adecuados para fondear, islas y ensenadas, además de localizar recursos básicos para la navegación y manutención de la tripulación como leña, agua y comida ${ }^{40}$.

A pesar de la contribución de estas expediciones a la generación de un cuerpo de conocimiento sobre la geografía austral, los cambios dentro del sistema de competencia imperial por el control del Pacífico motivaron nuevas exploraciones a la costa patagónica. En particular, la suscripción de la primera convención de Nutka entre España y Gran Bretaña gatilló un cuestionamiento de las autoridades coloniales hispanoamericanas, quienes requerían información acerca de la presencia de ingleses en los mares australes y un conocimiento más riguroso de la geografía de las costas patagónicas occidentales. Las nuevas expediciones fueron comandadas por Nicolás Lobato y José de Moraleda a principio de la década de 1790.

La expedición a cargo de Lobato estudió,

39 Martinic, 2004, op. cit., p. 113.

40 Ugarte, 1889. Diario del viaje de Don Cosme Ugarte a las costas occidentales de Patagonia 1767-1768. Anuario Hidrográfico de la Marina de Chile. Tomo XIV, 49-55, p. 51; Machado, 1889. Viajes del Piloto don Francisco Machado a los archipiélagos occidentales de Patagonia. Anuario Hidrográfico de la Marina de particularmente, el área de los archipiélagos de los Chonos y las Guaitecas. Zarpó el 7 de enero de 1792 desde Chiloé y tras tres meses en los canales patagónicos, regresó con importante información de esta geografía remota. José de Moraleda, por otro lado, condujo tres consecutivas expediciones a los canales durante los veranos de 1793, 1794 y 1795. Como se desprende de las instrucciones de estas expediciones, para las autoridades coloniales hispanoamericanas el espacio marítimo patagónico era pensado y valorado como un área de conexión entre el océano Atlántico y el Mar del Sur. Por ejemplo, se le encomienda a Moraleda poner atención al descubrimiento de cualquier canal que pudiera conectar estos dos océanos ${ }^{41}$. Asimismo, se concibe el conocimiento de este espacio como clave para la seguridad imperial, pero también como estratégico para la proyección del imperio en los mares australes. En este contexto, en sus instrucciones a Lobato, el gobernador de Chile Ambrosio Higgins insistía en la necesidad de advertir la presencia de ingleses en estas costas australes, reconocer y mapear esta zona escasamente cartografiada y evaluar la posibilidad de fundar un asentamiento o presidio ${ }^{42}$.

Estas expediciones, particularmente las conducidas por Moraleda, crearon una imagen más precisa de la navegación y de la geografía de este espacio marítimo. Ellos reconocieron, midieron, describieron y mapearon estos mares, generando nuevo conocimiento de la zona. Este cuerpo de conocimiento hidrográfico y geográfico fue registrado en sus diarios de navegación y también en la producción de diversas cartas esféricas y mapas específicos de la zona, los que circularon entre las autoridades de Chiloé, Santiago, Lima y la metrópoli hispana. Asimismo, al producir nuevo conocimiento, estas expediciones entraron en un diálogo directo con las realizadas anteriormente, evaluando, confirmando o criticando la información que ellas proveyeron. Ello, se ve representado, por ejemplo, en la crítica que hace Francisco Clemente

Chile, Tomo XIV, 57-149, p. 124; Urbina, 2018a. Fuentes para la Historia de la Patagonia Occidental en el periodo colonial. Segunda parte: siglo XVIII. Ediciones Universitarias de Valparaíso, Valparaíso, pp. 288-292.

41 Urbina, 2018a, op. cit., p. 345.

42 Ibidem, p. 310. 


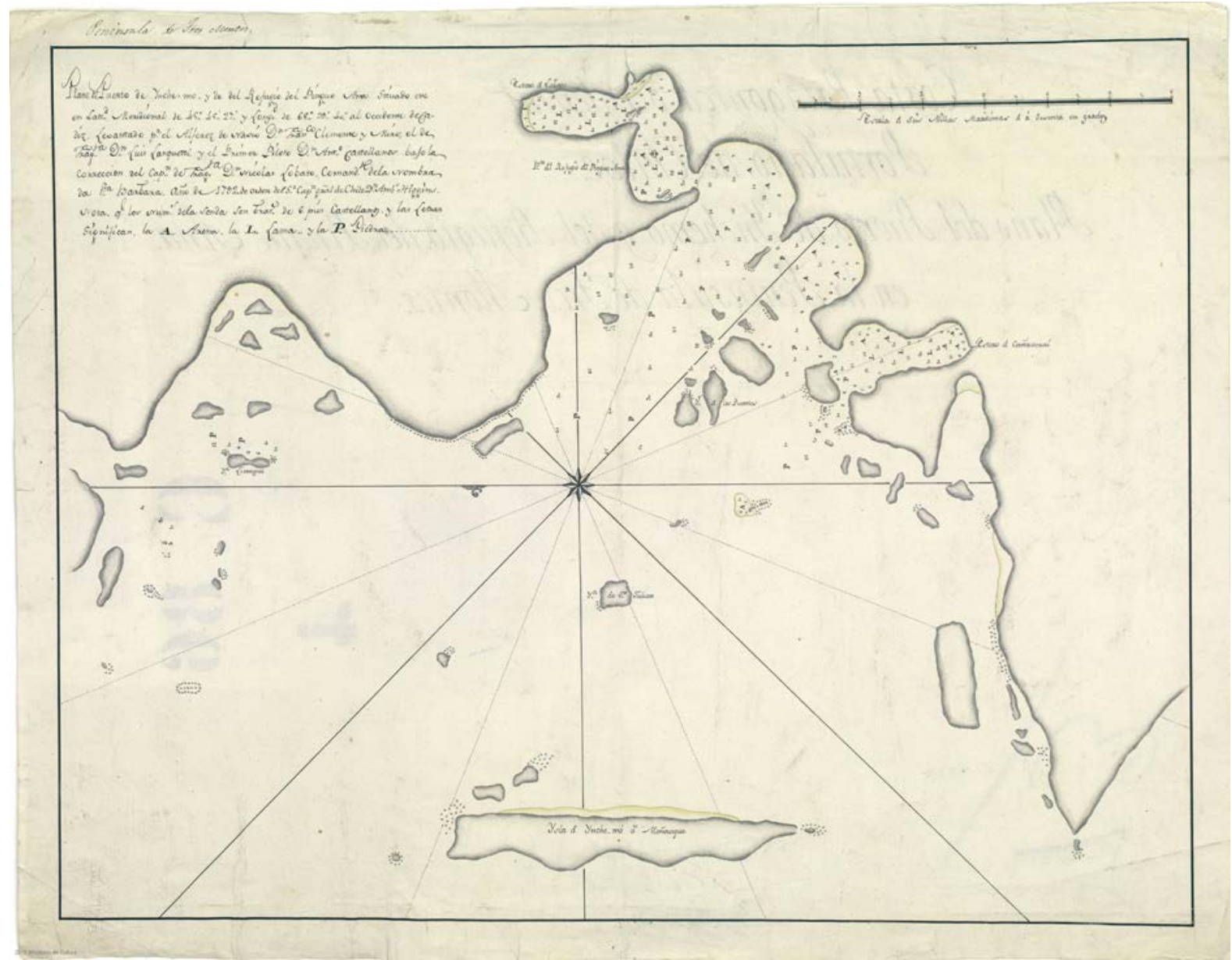

Fig. 5. Francisco Clemente y Miró, Plano del Puerto de Ynche-mo y del Refugio del Pingue Ana situado en Lattd Meridional de 4545'27" y Longd de 68³9'40" al occidente de Cádiz / levantado pr el Alférez de Navio Dn Franco. Clemente y Miró el de Fragta. Dn. Luis Lasquetti y el Primer Piloto Dn Anto.

Castellanos; bajo la corrección del Capn. de Fragta. Dn Nicolás Lobato, Comandte de la nombrada Sta Barbara. Año de 1792, de orden del Sr. Capitan gral. de Chile Dn. Ambrosio Higgins (1792). Museo Naval de Madrid MN 52-B-1.

y Miró, oficial que escribió el diario de navegación de la expedición comandada por Lobato. Clemente y Miró fue muy crítico de la información que los expedicionarios españoles habían desarrollado sobre el archipiélago de los Chonos y también de la dada por los marineros ingleses de la pink Anna que había naufragado en estas $\operatorname{costas}^{43}$. De este modo, se evidencia que la producción de conocimiento desarrollada por estas navegaciones no estuvo aislada de las redes de ciencia global; por el contrario, se inserta dentro de éstas, entrando en diálogo directo con ellas.
Asimismo, el material cartográfico producido da cuenta del proceso de estandarización de conocimiento hidrográfico dentro de las redes científico-navales del imperio español. De este modo, la expedición de Lobato logró construir distintos planos y cartas de la región. La Fig. 5, de la isla Inche, demuestra la rigurosidad y esfuerzo por desarrollar cartas siguiendo las convenciones de la cartografía de la época, determinando la ubicación de la isla con su latitud y longitud, lugares más aptos para fondear, señalando -al mismo tiempo- la profundidad de las bahías, la 
ubicación de bancos de arena y presencia de rocas y algas peligrosas para la navegación. De este modo, el análisis de la cartografía realizada por estas expediciones ayuda a evidenciar cómo los estándares de la ciencia hidrográfica global fueron adoptados en su cabalidad por los hidrógrafos que salieron desde Chiloé a fines del siglo XVIII. En este sentido, se debe destacar cómo la renovación de las ciencias navales influyó en la transformación y profesionalización de la actividad científica en Hispanoamérica.

La especialización de los pilotos y el impacto de las reformas de ciencia náutica se aprecian particularmente en la obra de Moraleda como hidrógrafo y cartógrafo. En su Carta Esférica de la Patagonia occidental (Fig. 6), éste realizó una descripción detallada de los principales elementos geográficos e hidrográficos de la región, nombrando cada uno de ellos. En la carta, el oficial español incluyó islas y bahías que anteriormente no habían sido registradas por la cartografía colonial, como por ejemplo las pequeñas islas cercanas al estero de Comau. Usando los datos referenciales de Malaspina, Moraleda cuidadosamente ubicó cada isla, bahía, ensenada y puerto. En sus anotaciones dentro de la carta, el piloto especificó que el espacio en blanco hacía referencia a la ausencia de investigaciones hidrográficas, por ello, no aparece representado en la carta. En este espacio también integró sus pensamientos y reflexiones acerca del territorio y mar patagónico. Así, remarcó que en el mar interior de la Patagonia occidental no había buenos puertos ni fondeaderos, que los esteros y ríos no eran útiles para la navegación (siendo solo los de Aysén, Comau y Palena navegables en piraguas), y que el suelo, tanto en las islas como en el continente, no era apto para el cultivo. De este modo, Moraleda generó una imagen muy negativa de la geografía patagónica, remarcando la manera en que este espacio no era útil ni para la explotación agrícola ni comercial, ni menos aún para la creación de asentamientos españoles.

El acento en los procesos de producción y circulación de conocimiento conlleva a analizar

44 Ibidem, p. 311.

45 Urbina, Reyes y Belmar, 2020. Canoeros en Chiloé: de facilitadores de las navegaciones españolas en los archipiélagos de los Chonos y de Guayaneco, a productores y comerciantes, 1567-1792. Chungara, 52(2). las prácticas y dinámicas sociales en las empresas científico-hidrográficas hispanoamericanas. Como lo demuestran las instrucciones que el gobernador de Chile Ambrosio Higgins dio al capitán Lobato, se consideraba como esencial el conocimiento de personas (locales) expertas en la geografía de la región ${ }^{44}$, quienes eran llamadas prácticos. Como se lee en los diarios de navegación, estos prácticos podían ser indios -generalmente de las comunidades chonos o caucahues- llamados indios prácticos, o podían ser chilotes, llamados genéricamente prácticos. Estos actores, siempre hombres tal como evidencia el reciente artículo de Urbina, Reyes y Belmar ${ }^{45}$, tenían el rol de guiar la navegación a través de los canales patagónicos, informar sobre lugares dónde fondear y encontrar recursos claves como agua, comida y madera. Por ejemplo, el diario de navegación de Francisco Clemente y Miró -oficial de la expedición de Lobatoda cuenta cómo estos actores daban indicaciones precisas dónde fondear y resguardarse, cuestión fundamental en una geografía como la de los canales patagónicos occidentales, donde el fondo marino no es profundo y las bahías tienden a tener vientos contrarios que dificultan la navegación ${ }^{46}$.

El diario de José de Moraleda también da luces acerca del rol cumplido por estos prácticos. En este documento se indica el número y nombre de los indios prácticos que integraban la expedición -Pedro Yaña, Manuel Tuba, Antonio y Luis Allupa-, además del pago que se les efectuaba -8 pesos mensuales- ${ }^{47}$. Moraleda, al igual que Clemente y Miró, confía en la experiencia y conocimiento de estos prácticos. Por ejemplo, en su recorrido desde las islas del archipiélago de los Chonos hacia la costa continental de Aysén, Moraleda confía la ruta a Pedro Yaña, quien poseía ganado en esta costa, y por ello conocía el camino hacia el lugar ${ }^{48}$. Yaña, no solo determinó la ruta, sino que también estudió las condiciones para efectuar la navegación, esperando el tiempo adecuado para atravesar los peligrosos canales ${ }^{49}$. Además de producir información sobre rutas de navegación conocidas, el diario de Moraleda demuestra el activo rol de

46 Urbina, 2018a, op. cit., pp. 318-319.

47 Ibidem, p. 348.

48 Ibidem, p. 366.

49 Idem. 
estos actores en la exploración de nuevos sitios. Por ejemplo, los indios prácticos acompañaron a los oficiales en la exploración del río Aysén.

La información producida por estos actores fue recogida y traducida a los estándares de la cartografía marítima tal como se demuestra en la Carta Esférica de Moraleda (Fig. 6), donde se presentan las islas y canales que fueron mostrados por los indios prácticos. Asimismo, esta información fue integrada en los informes y diarios de navegación producidos tanto por Clemente y Miró como por Lobato. Esto incluía anotar cuidadosamente la ruta seguida y advertir de la localización de lugares de fondeo y refugio. Tal como lo sugiere Brian Larking, esta forma de estandarización de conocimiento debe ser entendida como una forma de poder ${ }^{50}$. Al transferir los conocimientos de los prácticos al dispositivo de los diarios de viaje o a la carta marítima, se invisibiliza el aporte de estos actores al conocimiento hidrográfico de los mares del sur.

Por último, el análisis de estas expediciones permite un primer acercamiento a las redes regionales de producción de conocimiento hidrográfico en Hispanoamérica. Ya sea obedeciendo dictámenes generales emanados por la Corona o siguiendo sus propias iniciativas, las autoridades coloniales fueron activas promotoras $y$ organizadoras de expediciones de reconocimiento geográfico en el Pacífico austral. Tal como lo han argumentado Buschmann, Slack y Tueller, la decisión de realizar expediciones militares e hidrográficas al Pacífico básicamente correspondía a los virreinatos de Nueva España y Perú ${ }^{51}$. Así, por ejemplo, ante la amenaza de la presencia británica en el Mar del Sur, especialmente tras la circunnavegación del comodoro Byron, el virrey Amat impulsó la organización de empresas exploratorias a las costas patagónicas (Machado y Rius), así como también a las islas del Pacífico, particularmente a Rapa Nui o isla de Pascua (17701771) y dos viajes a Tahití (1772-1775). Con todo, a fines del periodo colonial, había emergido un cuerpo sustancial de conocimiento sobre el litoral del Pacífico americano, lo que incluía las hasta

50 Edwards et al. (2011). AHR Conversation: Historical Perspectives on the Circulation of Information. The American Historical Review, 116(5), 1392-1435, p. 1430 . entonces poco exploradas costas patagónicas occidentales. Ello queda también demostrado en la obra cartográfica de Andrés Baleato y José de Moraleda y su reconocimiento a las costas del virreinato.

Asimismo, este incremento en la producción de conocimiento del Pacífico americano fue de la mano con la institucionalización del mismo. Este es el caso de la formación de la Academia de Pilotos de Lima en 1793, que se convirtió en la primera institución de formación en ciencias náuticas del continente. El surgimiento de este tipo de instituciones muestra el interés local por generar conocimiento y prácticas navales útiles a los intereses regionales del virreinato peruano. Más aún, da cuenta del interés y necesidad de la élite local por formar oficiales en la navegación científica, instruyendo a los jóvenes estudiantes en distintas ramas como aritmética, geometría, trigonometría, cosmografía, dibujo, cartografía y el uso de instrumentos náuticos. Por ello, no debe extrañar que en el centro de esta institución se encuentren figuras claves de la hidrografía regional como los mencionados Moraleda y Baleato.

\section{CONCLUSIONES}

La importancia de las exploraciones científico-hidrográficas a los pasos interoceánicos del sudoeste ha sido poco resaltada por la historiografía, particularmente en comparación con la atención que ha recibido el paso por el noroeste americano. Luego de la circunnavegación de George Anson en la década de 1740, pero sobre todo después de los viajes del francés Bougainville y del capitán James Cook en los 1760s y 1770s, el conocimiento riguroso de los pasos interoceánicos fue considerado como estratégico para el desarrollo de los imperios europeos de ultramar. De este modo, el estrecho de Magallanes y el cabo de Hornos, así como también el litoral patagónico, volvieron a ser objeto de preocupación política por parte del imperio hispano. En este contexto, se organizaron expediciones desde la metrópoli española y desde el virreinato del Perú

51 Buschmann et al. (2014). Navigating the Spanish Lake: The Pacific in the Iberian World, 1521-1898. University of Hawai'i Press, Honolulu, p. 39. 


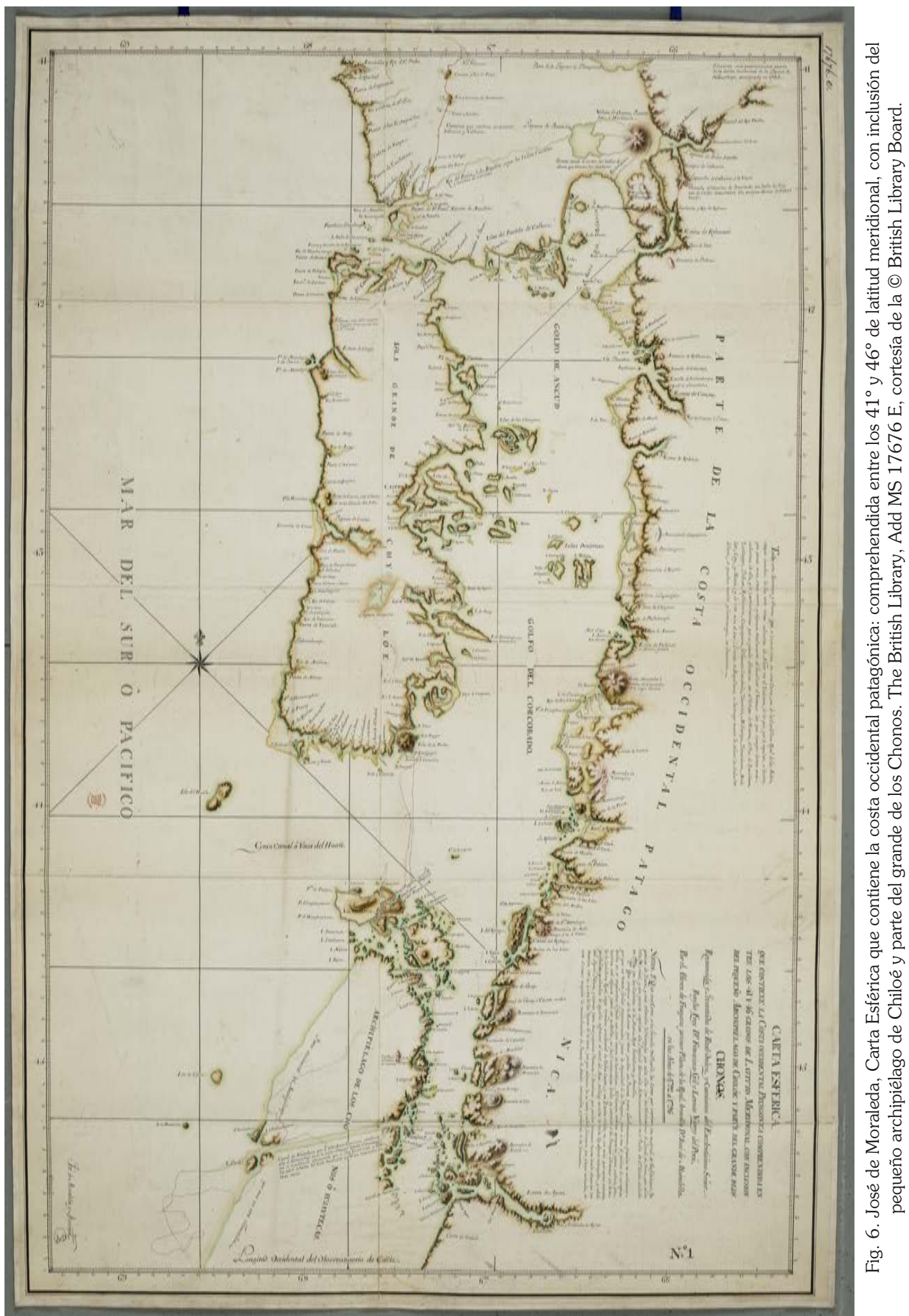


para crear conocimiento científico acerca de este espacio marítimo, aplicando las últimas técnicas de medición hidrográfica. De esta forma, una de las contribuciones de este artículo ha sido comprender la complementariedad de estas expediciones, entendiéndolas como parte de un esfuerzo en conjunto de la monarquía hispana por determinar la mejor ruta transoceánica.

El estudio de estas expediciones hidrográficas permite comprender la importancia de la hidrografía como actividad científica en un contexto de álgida competencia imperial entre las potencias europeas. En este sentido, el artículo ha buscado contribuir al estudio de la hidrografía, resaltando la instrumentalidad y utilidad de sus resultados para los intereses de la corona española. Estas expediciones convirtieron a este ambiente natural en un laboratorio hidrográfico al medir, describir y mapear esta geografía con los instrumentos y técnicas más avanzadas de la época. Asimismo, se ha enfatizado el carácter complementario de estas expediciones. Más aún, se ha postulado que estas empresas hidrográficas deben analizarse de manera conjunta con el fin de comprender los esfuerzos globales de la monarquía española por apropiarse política y simbólicamente de los pasos interoceánicos australes. Los resultados de éstas determinaron que la navegación interoceánica a través del estrecho de Magallanes no era una ruta apta, eficiente y segura, clausurando el uso de esta vía por cincuenta años, hasta que la navegación a vapor permitió reactivar el tránsito por ella. Como corolario, se mantuvo la vía del cabo de Hornos, privilegiándose la navegación por mar abierto para atravesar desde el Atlántico hacia el Pacífico. Adicionalmente, esto repercutió en la marginalización de la ruta de los canales interiores de la Patagonia occidental dentro de la navegación interoceánica, permaneciendo esta zona como periférica.

Asimismo, este artículo se ha centrado en el estudio de los procesos de producción y circulación de conocimiento en las empresas hispanas de reconocimiento de los pasos interoceánicos a fines del siglo XVIII. Más aún, las ideas emanadas del "spatial turn" en la historia de la ciencia han permitido cuestionar las narrativas nacionalistas que centran su análisis en los nuevos descubrimientos y aportes de estas empresas hidrográficas desde una perspectiva nacional. Se ha demostrado cómo estas expediciones entraron constantemente en diálogo con pasadas expediciones hidrográficas, usando, comentando y criticando la información y conocimiento emanados de ellas. De esta manera, la expedición de Córdoba estuvo informada fundamentalmente por las empresas españolas de Sarmiento de Gamboa y los hermanos Nodal, asî como también las expediciones contemporáneas de Cook y Bougainville. En particular, el estudio de la expedición liderada por Malaspina demuestra cómo estas empresas científicas hispanas fueron influidas por las investigaciones llevadas a cabo por otras potencias europeas, además de sustentarse en información producida en Hispanoamérica.

Por otro lado, el enfoque en los procesos de producción y circulación de conocimiento permite adentrarse en las dinámicas sociales dentro de las empresas hidrográficas. En particular, ha sido instrumental para relevar los distintos actores asociados a las exploraciones. De esta forma, se ha argumentado cómo las expediciones hispanoamericanas construían las rutas de navegación en base a los conocimientos y experticias de actores locales. Tanto las expediciones de Lobato como las de Moraleda hacia los canales patagónicos occidentales llevaron consigo como guías de navegación a expertos navegantes de la región, ya fueran marineros chilotes o personas de las comunidades chonas o caucahues. Estos guías, llamados prácticos, dirigieron la ruta de navegación, determinaron los lugares dónde recalar y refugiarse, dónde abastecerse de agua y otros recursos claves como madera, y aconsejaron frecuentemente a los comandantes de las expediciones sobre cómo y cuándo proceder con la navegación. Este enfoque, entonces, permite recentrar las narrativas de la historia de la ciencia al resaltar la importancia de actores no-europeos al conocimiento global de los mares australes. El conocimiento aportado por ellos fue apropiado por los líderes de la expedición, quienes posteriormente lo registraron en sus diarios de navegación, estandarizándolo dentro de los cánones de la ciencia hidrográfica contemporánea.

Adicionalmente, el estudio de la cartografía permite comprender cómo el conocimiento geográfico y cartográfico circuló y fue transferido 
entre los territorios americanos y entre éstos y la metrópoli. A partir de estos documentos se desprende que la construcción de conocimiento sobre la geografía de las costas australes no fue tan solo obra de expediciones metropolitanas, sino que en ella influyeron fuertemente expediciones organizadas por las autoridades coloniales. Por ejemplo, el mapa del ministro Lángara sobre las costas sudamericanas da cuenta de cómo los resultados de estas expediciones hispanoamericanas, caracterizadas muchas veces como locales, fueron integrados a la cartografía imperial. Al mismo tiempo, tal como se ha evidenciado en el artículo, la forma en que este conocimiento fue incorporado muestra, además, una asimetría de poder y procesos de apropiación de conocimiento, donde los resultados de la empresa metropolitana son presentados como nuevos, mientras que el conocimiento producido por los pares hispanoamericanos es invisibilizado.

Finalmente, el análisis de las expediciones a la Patagonia occidental permite un primer acercamiento a las redes regionales de conocimiento hidrográfico y cartográfico. Como se ha evidenciado, a fines del periodo colonial estaba emergiendo un conocimiento regional sobre el océano Pacífico, lo que comprendía desde las costas continentales de Sudamérica hasta las islas del Pacífico, principalmente Tahití. Asimismo, la importancia que adquirió la generación de conocimiento del Pacífico americano, particularmente en la zona de influencia del virreinato peruano, fomentó la necesidad de crear instituciones a nivel colonial -como la Academia de Pilotos de Lima- las que apoyaron sucesivas empresas de reconocimiento hidrográfico y cartográfico. En especial, el desarrollo de esta academia viene a demostrar el interés de la élite hispanoamericana por formar un cuerpo local de oficiales instruidos en las ciencias de la navegación, particularmente en ramos útiles para la gobernanza imperial como la cartografía y el pilotaje. El análisis de estas instituciones, entonces, representa un primer paso hacia la comprensión de las redes hispanoamericanas de conocimiento, y la manera que éstas sustentaron, formaron $y$ resguardaron formas particulares de conocer $y$ de hacer ciencia.

\section{AGRADECIMIENTOS}

Este artículo es resultado de la tesis doctoral financiada por Becas Chile, Comisión Nacional de Ciencia y Tecnología (CONICYT).

\section{BIBLIOGRAFÍA}

Achbari, A. (2015). Building Networks for Science: Conflict and Cooperation in Nineteenth-Century Global Marine Studies. Isis, 106(2), 257-282.

Álvarez, G. S. G. (2010). Las conexiones entre el pensamiento de Alejandro Malaspina y la representación visual de la expedición en la Patagonia (1789-1794). Magallania, 38(1), 5-18.

Anderson, K. (2018). Reading and writing the scientific voyage: FitzRoy, Darwin and John Clunies Ross. The British Journal for the History of Science, 51(3), 369-394.

Ardash, M. (2012). El Pacífico Hispanoamericano. Politico y Comercio En El Imperio Español (1680-1784). Ciudad de México: El Colegio de México.

Armitage, D. (2014). The International Turn in Intellectual History. En D. McMahon \& S. Moyn (Eds.), Rethinking Modern European Intellectual History (pp. 232-252). New York: Oxford University Press.

Armitage, D., \& Bashford, A. (2014). Pacific Histories. Ocean, Land, People. Basingstoke: Palgrave Macmillan.

Ballantyne, T. (Ed.) (2004). Science, Empire and the European Exploration of the Pacific. Aldershot: Ashgate/ Variorum.

Bernabeu, S. (1992). El Pacífico ilustrado: del lago español a las grandes expediciones. Madrid: Mapfre.

Burnett, D. G. (2009). Hydrographic Discipline among the Navigators: Charting an "Empire of Commerce and Science" in the Nineteenth-Century Pacific. En Akerman, J. (Ed.), The Imperial Map: Cartography and the Mastery of Empire. Chicago: University of Chicago Press.

Buschmann, R. (2014). Iberian Visions of the Pacific Ocean, 1507-1899. Basingstoke: Palgrave Macmillan.

Buschmann, R. F., Slack, E. \& Tueller, J. B. (2014). Navigating the Spanish Lake: The Pacific in the Iberian World, 1521-1898. Honolulu: University of Hawai'i Press.

Cañizares-Esguerra, J. (2001). How to write the history of the New World: histories, epistemologies, and identities in the eighteenth-century Atlantic world. Standford: Standfor University Press.

Cañizares-Esguerra, J., \& Breen, B. (2013). Hybrid Atlantics: Future Directions for the History of the Atlantic World. 
History Compass, 11(8), 597-609.

Cook, J. (1893). Captain Cook's Journal during his first voyage round the world made in H.M. Bark Endeavour 1768-1771. London: Elliot Stock.

Davids, K. (2015). Introduction: Bridging Concepts. Isis, 106(4), 835-839. Retrieved from JSTOR.

Day, A. (1986). Search for the Northwest Passage: An Annotated Bibliography. New York: Garland

Edney, M. (2019). Cartography. The ideal and its history. Chicago: The University of Chicago Press.

Edwards, P. N., Gitelman, L., Hecht, G., Johns, A., Larkin, B., \& Safier, N. (2011). AHR Conversation: Historical Perspectives on the Circulation of Information. The American Historical Review, 116(5), 1392-1435.

Estefane, A. (2005). La proyección nacional de una empresa imperial: la expedición Malaspina (1789-1794) en Chile republicano. Historia, 38(2), 287-326.

Hanisch, W. (1982). La isla de Chiloé, capitana de rutas australes. Santiago: Academia Superior de Ciencias Pedagógicas de Santiago.

Igler, D. (2013). The Great Ocean. Pacific Worlds from Captain Cook to the Gold Rush. New York: Oxford University Press.

Livingstone, D. N. (2005). Science, text and space: thoughts on the geography of reading. Transactions of the Institute of British Geographers, 30(4), 391-401.

Lois, C. (2014). Mapas para la Nación. Episodios en la historia de la cartografía argentina. Buenos Aires: Biblios Ed.

Maeso, J. (2005). Expediciones navales españolas a la Patagonia argentina durante el siglo XVIII, Madrid: UNED.

Maroto, M. (2005). Producing the Pacific. Maps and Narratives of Spanish Exploration (1567-1606). Amsterdam: Rodopi.

Martinic, M. (2004). Archipiélago Patagónico. La última frontera. Punta Arenas: Ediciones de la Universidad de Magallanes.

Matsuda, M. (2012). Pacific Worlds. A history of Seas, Peoples, and Cultures. Cambridge: Cambridge University Press.

McCook, S. (2013). Introduction. Isis, 104(4), 773-776.

Parker, K. (2018). Pepys Island as a Pacific stepping stone: the struggle to capture islands on early modern maps. The British Journal for the History of Science, 51(4), 659-677.

Pimentel, J. (1989). Malaspina y la Ilustración. Madrid: Ministerio de Defensa.

Pimentel, J. (1994). Ciencia y Política en el pensamiento colonial de Alejandro Malaspina (1754-1794).

Madrid: Universidad Complutense de Madrid.

Postnikov, A. (2004). The Search for a Sea Passage from the Atlantic Ocean to the Pacific via North America's Coast: On the History of a Scientific Competition. En Ballantyne (Ed.) Science, Empire and the European Exploration of the Pacific. Aldershot: Ashgate/ Variorum, 2004), 199-222.

Riera, E. (1988). Carlos III y el control del estrecho de Magallanes. La expedición del capitán de navío don Antonio de Córdoba (1785-1786). $2^{\circ}$ Congreso de Historia Militar. Colección ADALID (Tomo III. Comunicaciones II, pp. 101-112). Zaragoza: Servicio de Publicaciones del EME

Roberts, L. (2009). Situating Science in Global History: Local Exchanges and Networks of Circulation. Itinerario, 33(1), 9-30.

Rozwadowski, H. M. (2008). Fathoming the ocean: the discovery and exploration of the deep sea. London/ Cambridge, Mass: Belknap.

Sagredo, R. (2013). De la hidrografía imperial a la hidrografía nacional. Reconocimientos del Pacífico Sur. Siglos XVIII y XIX. Anuario de Estudios Americanos, 70(2), 509-556.

Sagredo, R. \& González, J.I. (Eds.). (2004). La expedición de Malaspina en la frontera austral del imperio español. Santiago: Editorial Universitaria y Centro de Investigaciones Diego Barros Arana.

Sagredo, R., \& Moreno, R. (Eds.). (2014). El Mar del Sur en la historia. Ciencia, expansión y poder en el Pacífico. Santiago: Dirección de Bibliotecas, Archivos y Museos.

Sanhueza, C. (Ed.). (2017). La movilidad del saber cientfico en América Latina. Objetos, prácticas e instituciones (siglos XVIII al XX). Santiago: Editorial Universitaria.

Secord, J.A. (2004). Knowledge in Transit. Isis, 95(4), 654672.

Sellés, M. (2000). Navegación astronómica en la España del siglo XVIII. Madrid: Universidad Nacional de Educación a Distancia.

Smith, J. W. (2018). To Master the Boundless Sea: The U.S. Navy, the Marine Environment, and the Cartography of Empire. Chapel Hill: University of North Carolina Press.

Urbina, X. (2010). La navegación por los canales australes en la Patagonia Occidental Insular en los siglos coloniales: la ruta del istmo de Ofqui. Magallania, 38(2), 41-67.

Urbina, X. (2013). Expediciones a las costas de la Patagonia Occidental en el período colonial. Magallania, 41(2), 51-84. 
Urbina, X. (2014). El frustrado fuerte de Tenquehuen en el archipiélago de los Chonos, 1750: La dimensión Chilota de un conflicto hispano-británico. Historia, 47(1), 133-155.

Urbina, X. (2016). La sospecha de ingleses en el extremo sur de Chile, 1669-1683: Actitudes imperiales y locales como consecuencia de la expedición de John Narborough. Magallania, 44(1), 15-40.

Urbina, X. (2017). La isla Madre de Dios (costa del Pacífico austral) en los siglos XVII y XVIII: circulación de la información e intereses geopolíticos de España $e$ Inglaterra. Vegueta, 17, 545-567.

Urbina, X. (2018a). Fuentes para la Historia de la Patagonia Occidental en el periodo colonial. Segunda parte: siglo XVIII. Valparaíso: Ediciones Universitarias de Valparaíso.

Urbina, X. (2018b). Los "papeles de Londres" y alertas sobre ingleses. Chiloé y las costas de la Patagonia Occidental ante los conflictos entre España e Inglaterra: siglos XVII y XVIII. Mélanges de la Casa de Velázquez, 48(2), 235-264.

Urbina, X., Reyes, O., \& Belmar, C. (2020). Canoeros en Chiloé: de facilitadores de las navegaciones españolas en los archipiélagos de los Chonos y de Guayaneco, a productores y comerciantes, 1567-1792. Chungara, $52(2)$.

Vázquez de Acuña, I. (1998). La expedición científico-política del Capitán de Navío Don Alejandro Malaspina (17891794). Boletín de la Academia Chilena de la Historia, 65(108), 133-174.

Vázquez de Acuña, I. (2004). Las exploraciones del Estrecho de Magallanes por el capitán de navío don Antonio de Córdoba y Lasso de la Vega. Su primer viaje (17851786); su segundo viaje (1788-1789). Revista de Historia Naval, 84, 7-26.
Williams, G. (1997). The Great South Sea. English Voyages and Encounters 1570-1750. New Haven: Yale University Press.

\section{FUENTES}

Byron, J. (1769). Viage del Comandante Byron alrededor del mundo, hecho últimamente de orden del almirantazgo de Inglaterra. Madrid: Casa de don Francisco Mariano Nipho.

Machado, F. (1889). Viajes del Piloto don Francisco Machado a los archipiélagos occidentales de Patagonia. Anuario Hidrográfico de la Marina de Chile, XIV, 57-149.

Ugarte, C. (1889). Diario del viaje de Don Cosme Ugarte a las costas occidentales de Patagonia 1767-1768. Anuario Hidrográfico de la Marina de Chile, XIV, 49-55.

Vargas Ponce, J. de (1788). Relación del último viaje al Estrecho de Magallanes de la fragata del S.M. Santa María de la Cabeza en los años de 1785 y 1786. Extracto de todos los anteriores desde su descubrimiento impresos y MSS y noticia de los habitantes, suelo, clima y producciones del Estrecho. Madrid: viuda de Ibarra, Hijos y Compañía.

Vargas Ponce, J. de (1793). Apéndice a la Relacion del viage al Magallanes de la fragata de guerra Santa María de la Cabeza, que contiene el de los paquebotes Santa Casilda y Santa Eulalia para completar el reconocimiento del estrecho en los años de 1788 y 1789. Madrid: Imprenta de la viuda de D. Joaquín Ibarra.

Vargas Ponce, J. de (1820). Voyage of discovery to the Strait of Magellan: with an account of the manners and customs of the inhabitants, and of the natural productions of Patagonia undertaken, by order of the King of Spain. London: Phillips. 